\title{
Dive Mechanic: Bringing 3D virtual experimentation to elite level diving using the Workspace workflow engine
}

\author{
$\underline{\text { R. C. Z. Cohen }}^{\text {a }}$, S. M. Harrison ${ }^{\text {a }}$, P. W. Cleary ${ }^{\text {a }}$ and M. Bolger \\ ${ }^{a}$ CSIRO Data61, Clayton, Victoria, Australia \\ Email: Raymond.Cohen@,csiro.au
}

\begin{abstract}
Dive Mechanic is a custom 3D software application for modelling elite level diving. It is designed to assist coaches, athletes and sports biomechanists in understanding, visualising and improving diving performance for both platform and springboard diving. This application was developed by research scientists within CSIRO Data61 and was built upon the Workspace workflow engine. Biomechanics algorithms were developed within this graphical workflow environment and an intuitive graphical user interface (GUI) was developed to make the software accessible to end users. Workspace also simplified the packaging of Dive Mechanic by providing an installer and access to software licensing controls. This paper outlines how the Workspace workflow engine assisted in the process of both developing scientific algorithms and encapsulating them within a commercial product offering.
\end{abstract}

Dive Mechanic was developed by CSIRO for use by Diving Australia as part of a larger project to investigate diving technique and injury risk. The aerial dynamics portion of the modelling was identified as sufficiently computationally-cheap to facilitate an interactive software solution. Dive Mechanic uses personalised body and motion data based on laser scans of the athletes and markerless motion digitisation from multiple camera video of the athletes performing dives. It employs a fast and detailed human biomechanics motion engine specifically developed for the application that allows diver technique to be modified interactively by coaches in order to explore technique or synchronisation improvements. Its GUI is powerful yet sufficiently simple and intuitive so as to provide a very positive user experience for non-IT diving experts. Dive Mechanic's virtual experimentation offers advantages over physical experimentation in that it can help avoid injury and having divers training possibly worse techniques whilst experimenting. Dive Mechanic was used by coaches of the Australian Olympic diving squad in preparation for the Rio 2016 Olympics.

The Workspace workflow engine was well suited to the development of Dive Mechanic. The first stage was to elicit requirements from Diving Australia and to formulate software specifications. Computational modelling of diving fits naturally within the workflow paradigm with the inputs of a physical diver model, diver technique and takeoff conditions being processed to output the complete diver aerial motion which feeds through to a visualisation workflow. From these specifications, the software architecture was determined in terms of functional blocks in a computational workflow and corresponding features in the user interface. Initial development involved creating the biomechanical file format readers, data structures, widgets and unit testing operations. Critical widgets for software usability including a kinematics modification widget and a simplified 3D OpenGL widget were then created. Next, the dynamics engine was developed and tested. Important features in the overall workflow were then connected with UI elements to produce the Dive Mechanic software. Incremental development of the workflow and the ability to easily inspect and interrogate data locally in the workflows using the same widgets used in the final product simplified software debugging and troubleshooting. Additionally the inherent modular nature of workflow operations maximised the reuse of existing operations and improved design of new reusable workflow operations by computational scientists.

Keywords: Workflows, computational modelling, software engineering, diving, biomechanics 


\section{INTRODUCTION}

Diving Australia (DA) is the authority for competitive diving in Australia and have expert understanding of the current state of coaching and learning for elite diving worldwide. Through work with CSIRO, DA identified that virtual experimentation with individual athlete technique could be a novel and effective activity for improving dive performances. Dive Mechanic is a software tool that was specifically designed to facilitate physically realistic and near real time simulation of dive performances to enable virtual experimentation. The needs of the software identified included the use of detailed computational biomechanical models of individual athlete anthropometry and technique; markerless motion capture of diving performances; the ability for nontechnical users to experiment with simulation inputs; tools for the analysis of the three-dimensional diving kinematics that are predicted by the simulation; and ability to help lower level regional athletes by providing new understanding developed from analysis of technique used by elite athletes.

\section{OVERVIEW OF SOFTWARE DEVELOPMENT PROCESS IN WORKSPACE}

Dive Mechanic was developed using CSIRO Workspace (Bolger et al., 2015, 2016; Cleary et al., 2015), which is a cross-platform workflow and application development framework designed to enhance productivity, enable collaboration and software reuse, and to enable the commercialisation of software products. Workflows are a network of operations connected together (example shown Figure 1a) with directed data flows and order of execution that can be used for any type of scientific computing application. Both workflows and operations are modular by design and therefore re-deployment of IP across projects or between collaborators is straightforward and robust. Because common data types are used across the developer base, operation outputs can be further analysed, visualised, or written to file with minimal requirements for extra work. Workflows can easily be combined with a graphical user interfaces (GUIs) to be deployed as standalone compiled applications. Each individual Workspace operation is written using $\mathrm{C}++$ and the Qt framework and can be incorporated into shareable plugins that can be the basis of many different workflows. The development of Dive Mechanic using workflows in the Workspace platform was made more efficient through the ability to visually and robustly test and experiment with overall design using the following procedure:

1. Identification and development of the required unit operations (File IO, model set up, simulation, post processing)

2. Construction of workflows that use these operations to perform key activities

3. Analysis of software usability, speed and accuracy and of model outputs using in-built widgets

4. Iterative improvement of operations and workflows to improve usability, interactivity and accuracy

5. Deployment of workflows in the form of a licensed standalone compiled software application to stakeholders

6. Further iterative improvement of software based on feedback from stakeholders

The operations and widgets developed are sharable via a Bio-Mechanic plugin for Workspace which include file readers and writers, pre-processing, dynamics solvers and post-processing.

Dive Mechanic addresses the requirements of Diving Australia in a single integrated package through a number of unique features:

- Simple and intuitive GUI.

- Easily relatable visually realistic diver models and environments.

- Highly detailed and accurate representations of individual athletes and diving physics.

- Ability to interactively change the diver technique and immediately see the consequences on dive performance.

- Augmentation of useful quantitative data, including three-dimensional view with diver trajectory, arrows showing velocities, principal moments of inertia and the centre of mass.

- Ability to show multiple divers for analysis of synchronised diving - the focus of the Olympic campaign.

This paper details the development of Dive Mechanic and how its development was enabled with the use of the Workspace platform.

\section{DIVER DIGITAL MODEL DEVELOPMENT}

Diving Australia identified two pairs of synchronized springboard female divers with potential medal prospects at the Olympics who would benefit from use of Dive Mechanic. Accurate biomechanical simulation of their performances required detailed models of the athlete's body shape and dive technique. Use of marker based motion capture systems was deemed impractical in the aquatic environment. Consequently a markerless motion 
capture process suitable for diving was developed based on multi-camera video footage of the athletes diving and laser scans of their body shapes.

The motion capture process required video cameras to be calibrated. The OpenCV library contains general functions for calibrating cameras so these were wrapped inside Workspace operations in a shared OpenCV plugin. A workflow was developed (Figure 1Error! Reference source not found.a) for carrying out a calibration and it became evident that a graphical widget was required for marking up landmarks within stills from each camera view. The process was commonly required across different projects so the workflow had a simple GUI developed and was put inside a standalone application (Figure 1Error! Reference source not found.b). The diving trials were performed in the diving pool at the Brisbane Aquatic Centre and were filmed by a system of five fixed and synchronised COP Security cameras (COP Security Corp., Taiwan) as shown in Figure 2. Measurements of the pool arena, diving platforms and springboards were made to determine the world coordinates $(x, y, z)$ of visible landmarks. The application was then used to mark up images with these known landmarks (red crosses in Figure 3). Additionally features that were simultaneously visible from multiple viewpoints (e.g. diver finger tips from multiple camera angles) with unknown world coordinates were marked up and tagged with unique identifiers (yellow crosses). The software then used these sets of landmark data to perform an iterative calibration which provided the intrinsic (focal length and lens distortion) and extrinsic (position and orientation) properties of each camera. The widgets within the software GUI (Figure 1Error! Reference source not found.b) allowed the user to manipulate the parameters of the calibration in the underlying workflow (Figure 1Error! Reference source not found.a) and to automatically have the workflow/calibration be updated.

The athletes considered in this study were laser body scanned using a VITUS Smart XXL machine (Human Solutions GmbH, Kaiserslautern, Germany) to generate a static surface mesh as shown in Figure 4a. The laser body scan was then rigged to a simplified skeletal structure which enabled rotation of the joints to naturally deform the surface mesh (Figure $4 \mathrm{~b}$ ) utilising the commercial animation package Maya (Autodesk Inc., San Rafael, CA, USA). The model divers were then manipulated to match each frame of video footage for all cameras simultaneously (Figure 5). The resultant diving biomechanical models were suitable for use in the Dive Mechanic software. Further details of the markerless motion capture process are available in Harrison et al. (2016).

(a)

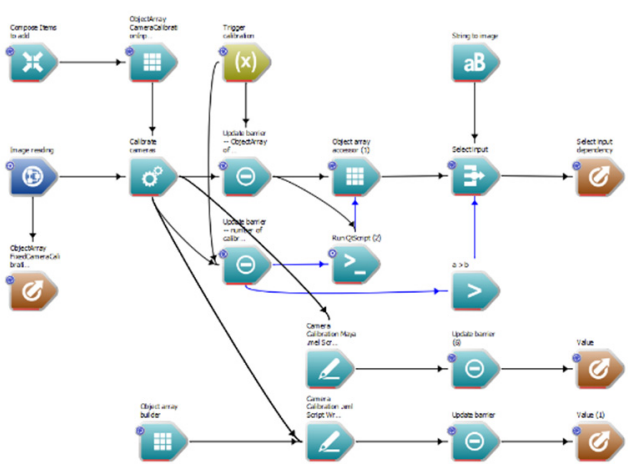

(b)

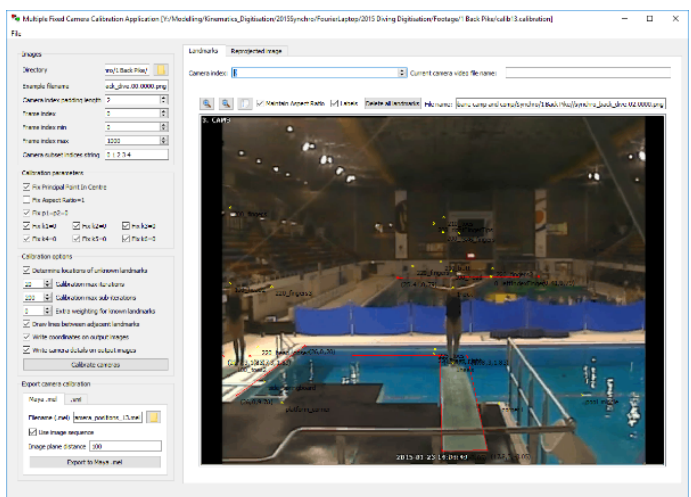

Figure 1. Multiple fixed camera calibration software (a) underlying workflow, and (b) GUI.

(a)

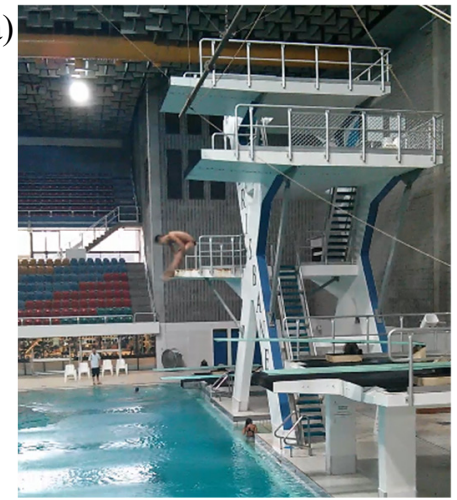

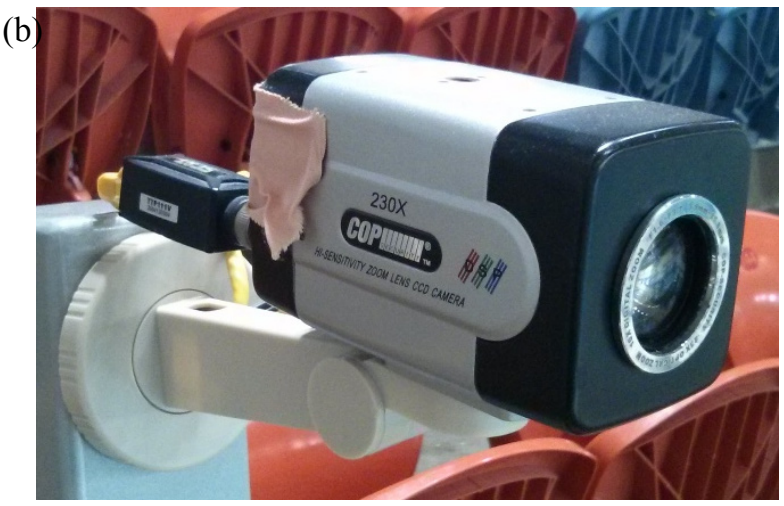

Figure 2. Brisbane Aquatic Centre (a) diving platforms and springboards, and (b) COP video camera. 

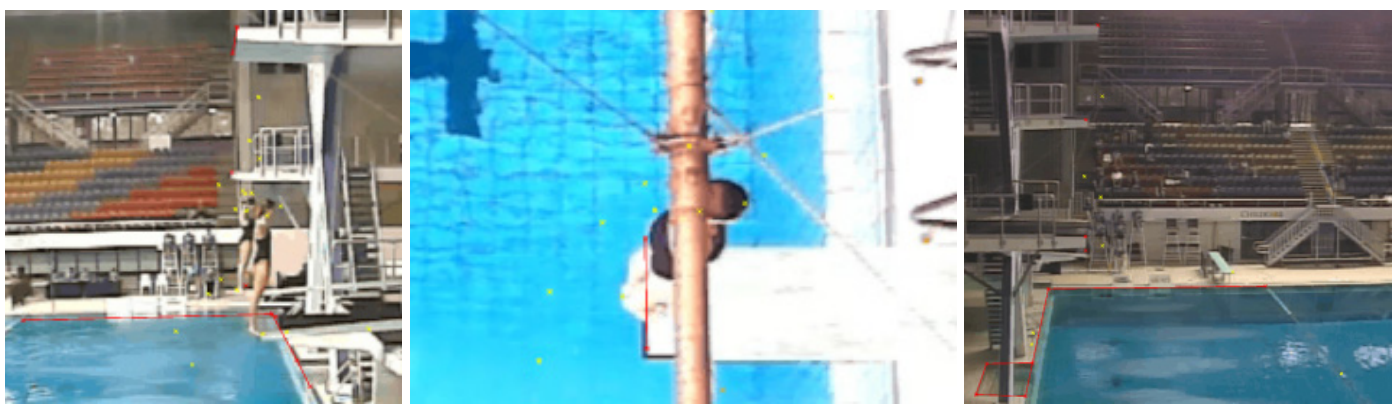

Figure 3. Cropped stills from each of the fixed video cameras with marked up landmarks. Red crosses indicate landmarks with known world coordinates $(x, y, z)$. Yellow crosses indicate features that are simultaneously visible in multiple views but don't have known world coordinates. Red lines are drawn between adjacent landmarks.

(a)

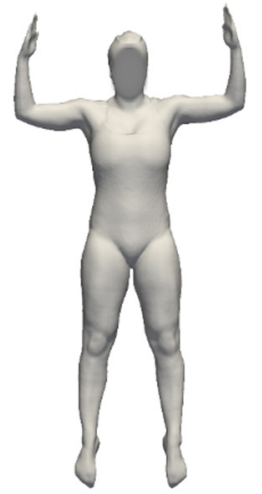

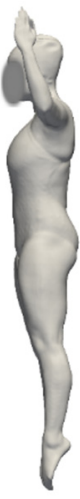

(b)

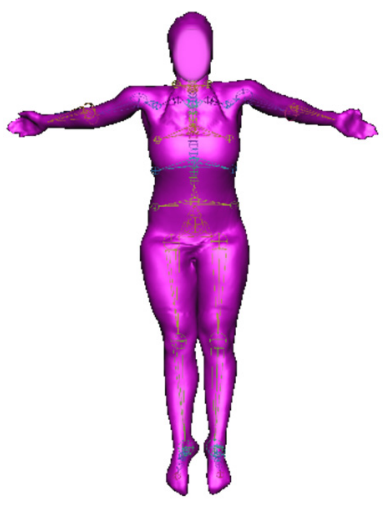

Figure 4. Biomechanical model of the diver (a) laser body scan and (b) rigged model posed to match part of the dive sequence
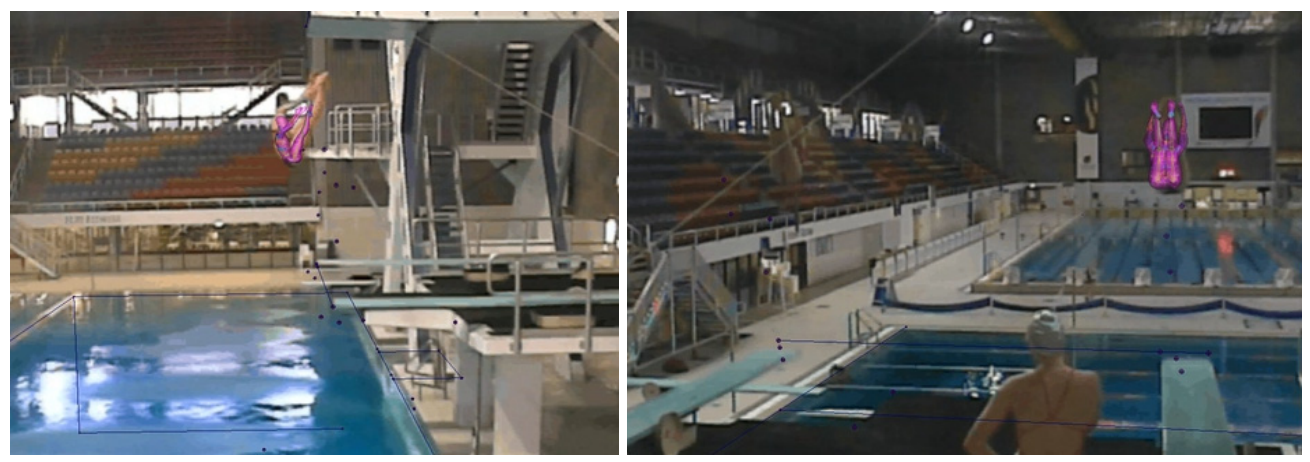

Figure 5. Example frame of the digitization process where the diver model (pink) pose is manipulated until it overlays the athlete exactly in the multiple camera views. In the leftmost figure the far diver is partially obscured by the nearer driver.

\section{DIVE MECHANIC DEVELOPMENT}

The first stage in developing the Dive Mechanic software was to create data types, widgets and readers for the biomechanical model. Testing each of these basic operations was done by putting the operations into individual workflows and verifying the outputs as displayed in the graphical widgets (Figure 6Error! Reference source not found.). Automated regression testing involved adding additional checks on the operation outputs within the workflow. 


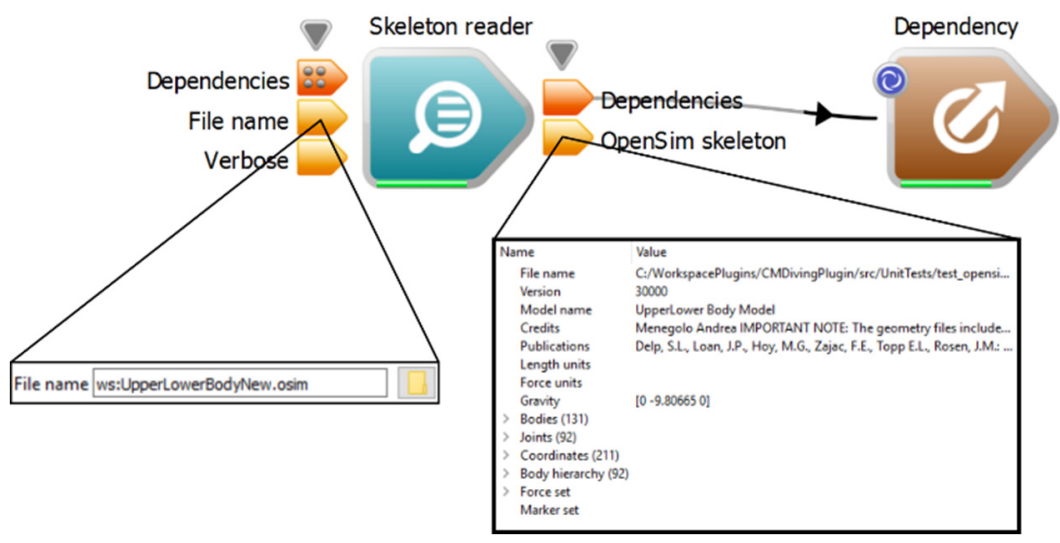

Figure 6. Skeleton reader operation with widgets shown for the file name input and the skeleton output.

The dynamics of the diver centre of mass depends on the temporal changes of joint angles during the dive performance. Mesh deformation algorithms were used to map the time varying joint angles to the deformed shape of the athlete skin mesh. Algorithms to calculate the volume and moments of inertia of the athlete mesh for each frame of input kinematics were then developed and tested. The key user requirements for the Dive Mechanic was the ability for the user to modify the diver take-off conditions and timing of key kinematics events. Based on these understood use cases, the data types and widgets were designed and tested within the Workspace environment (Figure 7Error! Reference source not found.). Next the dynamics solver operation was developed which enabled the calculation of the diver motion through the air depending on the take-off conditions and specified digitised diver technique (Figure 8Error! Reference source not found.a). The dynamics solver operation predicts the full motion of the diver throughout the dive. These simulation outputs were connected to the Workspace OpenGL 3D rendering operations to display the diver motion in a graphical representation. The basic functionality of Dive Mechanic was then complete and was able to be interrogated by software developers using the Workspace editor environment by inspecting variables used within the workflow.

Once the required capabilities were demonstrated in workflows, a dedicated GUI was constructed in order to create a standalone application (Figure 8Error! Reference source not found.b,c). The GUI was built using Qt Designer (as is usual for Workspace applications) which enabled easy arrangement of menus, toolbars, standard widgets and custom widgets within a main window. Connecting up the workflow to the GUI was done by the usual Workspace development method of clicking on each Workspace workflow input/output and then dragging the mouse to the corresponding Qt Designer widget. This is a simple to use method for connecting the graphical and workflow layers of the application and is accomplished without any code needing to be written. The creation of the standalone Dive Mechanic application required the creation of thin $\mathrm{C}++$ main program (with the assistance of a Workspace wizard). This loads the GUI and workflow, connects them together and then initiates the workflow operation.

The rest of the development process consisted of refining the software interface to make it as simple to use and as visually appealing as possible (Figure 9a,b). The interface consists of a large OpenGL viewport which the user can click on to rotate the camera view, a playback widget to scrub back and forth through the dive, and widgets for modifying the dive. A large database of approximately 60 dives necessitated the development of a dive selection widget which enables the user to filter the dive list by a number of criteria (Figure 9c). Problems with slow rendering speed were resolved by reducing the level of detail with reduction algorithms to the diver meshes. An aesthetic textured diving arena (based on the Brisbane Aquatic Centre) was added to improve the appeal of the software to diving coaches and athletes who hadn't previously used scientific modelling software.

The final steps for the software development were to add software licensing and an automated installer. Software licensing was added using the CSIRO L4TM library which prevents the software from starting without a valid license file. A Workspace workflow was created which can make installer packages for three platforms - Microsoft Windows, MacOS and Linux (Figure 8Error! Reference source not found.d). If Windows is the target platform, then Workspace creates a Nullsoft based installer. The Dive Mechanic software went through a number of iterations in which the prototypes were reviewed and evaluated by stakeholders with feedback provided back to the developers. This enabled the software to be fine-tuned prior to being distributed to the diving coaches. The dive database was included with the software, covering the year in the lead up to the Rio Olympics. 
(a)

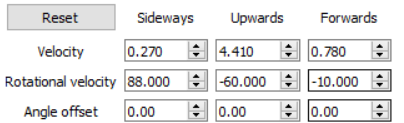

(b)

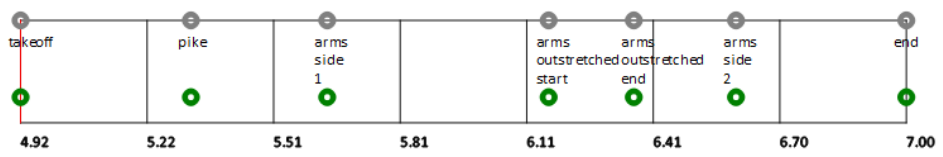

Figure 7. Custom widgets for controlling the actions of the diver including, (a) modification of the diver's takeoff translational velocity and rotational velocity along with an angle offset, and (b) modification of the timing of key events during the dive.

(a)

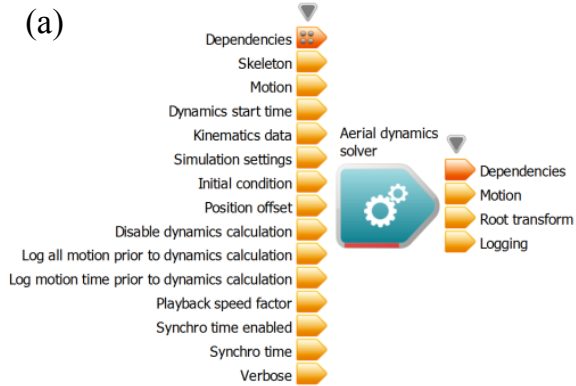

(c)

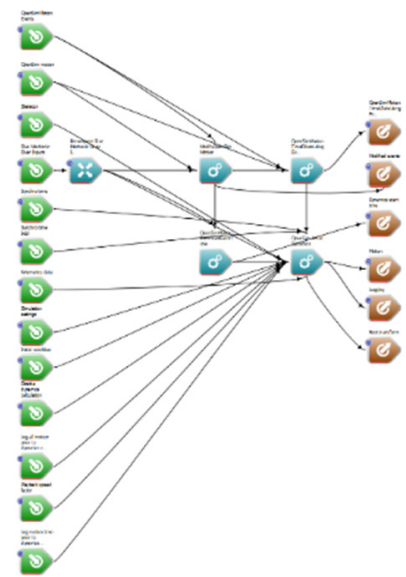

(b)

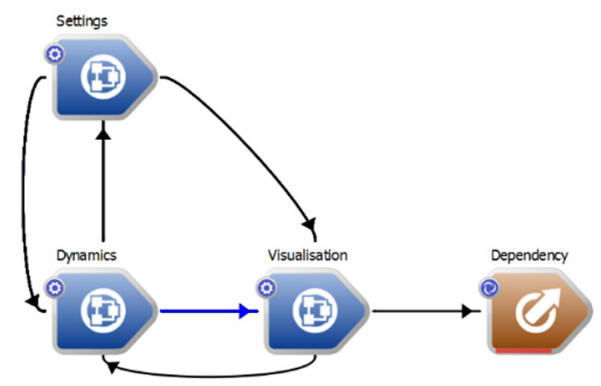

(d)

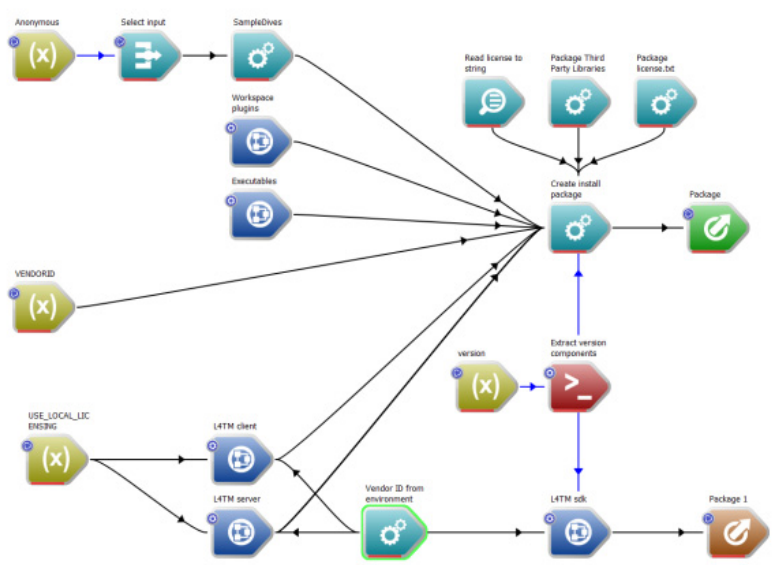

Figure 8. Dive Mechanic workflow elements. Green blocks are inputs, brown blocks are outputs, cyan blocks are operations and blue blocks are nested workflows. The segments show directed connections between blocks that indicate data flow and block dependencies. (a) Diving aerial dynamics solver showing the inputs (left) and outputs (right). (b) Top level workflow. (c) Example nested workflow. (d) Workflow which builds an installer executable.

\section{CONCLUSIONS}

Dive Mechanic was developed by research scientists using the Workspace workflow engine. This framework ensured the developers created highly modular and reusable elements (operations, data types and widgets). The workspace editor facilitates testing of operations with inspection of often complex inputs and outputs using suitable widgets. The Workspace editor also enabled the Dive Mechanic workflows to be developed and tested prior to the existence of a GUI. The attachment of the GUI widgets to the workflow was simple and didn't require any written code. Tailoring the user interface took a few internal and external iterations to optimise usability and performance. The cross platform software was able to be delivered to the client with an automated installer and licensing instructions.

Dive Mechanic's overall impact for Diving Australia was to allow new evidence based experimentation and communication facilities to be used by the woman's Olympic diving team - providing a technological edge over the rivals. Usage of Dive Mechanic can be at both the elite competitive level and all the lower-level regional organisations. Additionally the underlying human dynamics models can be readily used in similar applications for other sports. The use of Workspace facilitates extensive re-use of the underlying biomechanics operations for these different applications. 


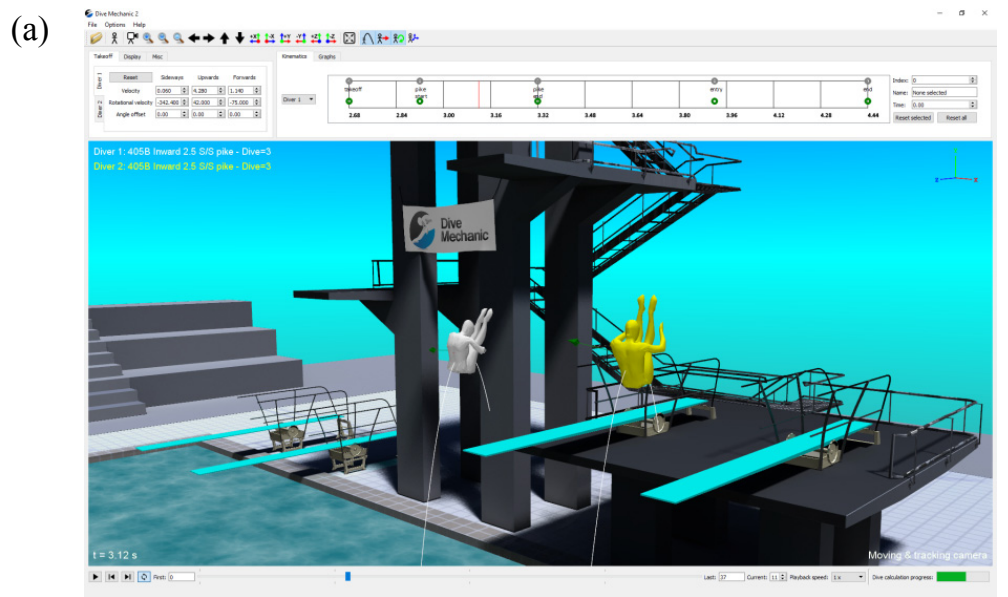

(b)

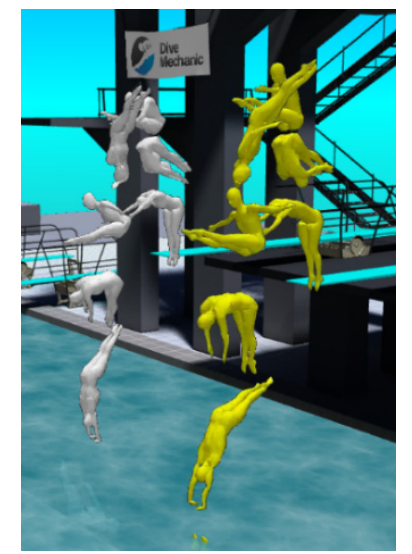

(c)

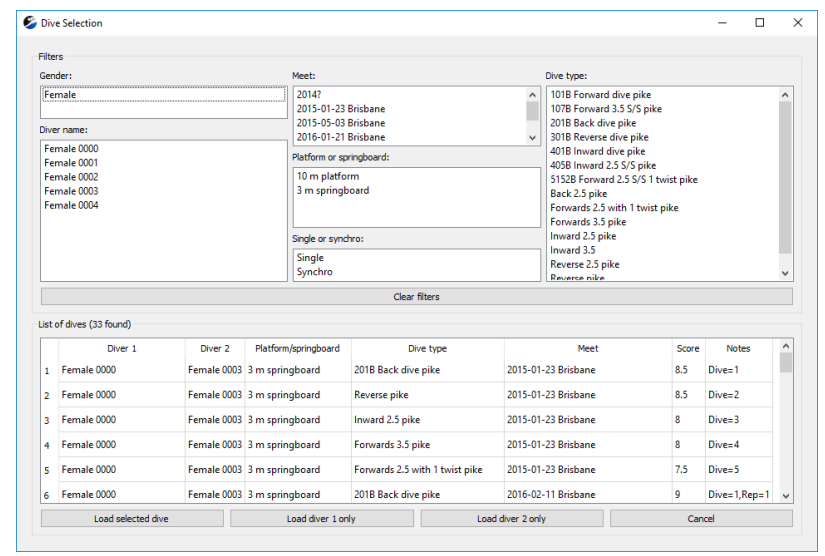

Figure 9. Dive Mechanic interface. (a) Screenshot of the Dive Mechanic software in action. Takeoff conditions can be modified for each diver using the tabs in the top-left of the screen whilst timing of kinematic events can be modified using the timeline widget above the 3D viewport. (b) Composition of a sequence of video frames showing a pair of synchronized divers completing a forward $2.5 \mathrm{~S} / \mathrm{S} 1$ twist pike.

(c) Diver selection widget.

\section{ACKNOWLEDGMENTS}

The work carried out in this project was funded by Australian Institute of Sport and in partnership with Diving Australia. The authors acknowledge the efforts of Valerie Beddoe in coordinating this project, filming diving trails and having athletes laser body scanned.

\section{REFERENCES}

Bolger, M., Cleary, P. W., Hetherton, L., Rucinski, C., Thomas, D., \& Watkins, D. (2015). Workspace: Scientific Workflows and Applications for multiple Environments. Presented at the eResearch Australasia.

Bolger, M., Cleary, P. W., Hetherton, L., Rucinski, C., Thomas, D., \& Watkins, D. (2016). Workspace: a fast and low cost methodology for delivering commercial applications based on Research IP. Presented at the eResearch Australasia.

Cleary, P. W., Thomas, D., Bolger, M., Hetherton, L., Rucinski, C., \& Watkins, D. (2015). Using Workspace to automate workflow processes for modelling and simulation in engineering. Presented at the $21 \mathrm{st}$ International Congress on Modelling and Simulation, Gold Coast.

Harrison, S. M., Cohen, R. C. Z., Cleary, P. W., Barris, S., \& Rose, G. (2016). A coupled biomechanicalsmoothed particle hydrodynamics model for predicting the loading on the body during elite platform diving. Applied Mathematical Modelling, 40(5-6), 3812-3831. 